\title{
Análise dos Testes de Vitalidade Fetal e dos Resultados Perinatais em Gestações de Alto Risco com Oligoidrâmnio
}

\author{
Analysis of Fetal Well-being and Perinatal Outcome in the High-risk \\ Pregnancies Complicated by Oligohydramnios
}

Roseli Mieko Yamamoto Nomura, Rossana Pulcineli Vieira Francisco, Seizo Miyadahira, Marcelo Zugaib

\section{RESUMO}

Objetivos: analisar, em gestações de alto risco com diagnóstico de oligoidrâmnio, os resultados dos testes de avaliação da vitalidade fetal e os resultados perinatais.

Métodos: foram selecionadas retrospectivamente 572 gestações de alto risco com diagnóstico de oligoidrâmnio, caracterizado por ILA inferior ou igual a 5,0 cm. Destas, 220 apresentavam diagnóstico de oligoidrâmnio grave (ILA $\leq 3,0 \mathrm{~cm}$ ). Os testes de avaliação da vitalidade fetal incluiram: cardiotocografia anteparto de repouso, perfil biofísico fetal (PBF) $e$ dopplervelocimetria das artérias umbilical e cerebral média. Não foram incluídas as gestações múltiplas, com anomalias fetais e rotura prematura de membranas.

Resultados: o grupo de gestantes com diagnóstico de oligoidrâmnio grave (ILA $\leq 3 \mathrm{~cm}$ ) apresentou cardiotocografia anteparto suspeita ou alterada em 23,2\% dos casos, PBF alterado em 10,5\%, dopplervelocimetria da artéria cerebral média com sinais de centralização (54,5\%), recém-nascidos pequenos para a idade gestacional (32,7\%) e liquido amniótico meconial (27,9\%). Estes valores foram significativamente mais elevados do que os do grupo com ILA entre 3,1 e 5,0 cm. Este grupo apresentou cardiotocografia anteparto suspeita ou alterada em 14,9\% dos casos, PBF alterado em 4,3\%, dopplervelocimetria da artéria cerebral média com sinais de centralização em 33,9\%, recém-nascidos pequenos para a idade gestacional em 21,0\% e líquido amniótico meconial em 16,8\% dos casos.

Conclusões: a caracterização da gravidade do oligoidrâmnio permite discriminar, nas gestações de alto risco, os casos que se associam a pior resultado perinatal.

PALAVRAS-CHAVE: Líquido amniótico. Oligoidrâmnio. Complicações da gravidez. Resultados perinatais.

O oligoidrâmnio é conceituado como diminuição na quantidade de liquido amniótico (LA), e a sua incidência é estimada entre 0,5 a $5,5 \%$ da gestações $^{1,2}$. Considera-se oligoâmnio situações em que o volume de LA é inferior a 300 ou 400 $\mathrm{mL}$, embora existam dificuldades para a estimativa exata deste volume durante a gravide $z^{1}$.

O oligoidrâmnio, quando relacionado à in-

Setor de Vitalidade Fetal - Clínica Obstétrica do Hospital das Clínicas da Faculdade de Medicina da Universidade de São Paulo

Correspondência:

Roseli Mieko Yamamoto Nomura

Rua General Canavarro, 280 - Bairro Campestre

09070-440 - Santo André - SP

Tel: (11) 4991-2481 tel: (11) 4221-8778 Fax: 4221-8752

e-mail: roseli.nomura@terra.com.br

Home Page: http://www.hcnet.usp.br/ob/ suficiência placentária, apresenta como mecanismo fisiopatológico, a redução gradativa da produção de $\mathrm{LA}^{3}$, provocada pelos fenômenos adaptativos desencadeados pela hipoxemia fetal crônica. Subseqüente ao déficit nutritivo, o regime de hipoxemia provoca resposta circulatória fetal, com centralização da circulação. Desta forma, os mecanismos cardiocirculatórios regulam a redistribuição do débito cardíaco fetal com aumento do fluxo sangüineo destinado aos órgãos nobres (cérebro e coração) em detrimento de outros órgãos como os rins e pulmões ${ }^{4}$. A diminuição gradativa do volume de LA torna o cordão umbilical suscetível a compressões ${ }^{5,6}$ e, em graus extremos de oligoidramnia, isto pode ser fator determinante do óbito fetal. 
O aprimoramento da ultra-sonografia e seu uso rotineiro nas gestações de alto risco possibilitaram o desenvolvimento de várias técnicas para avaliação do volume de líquido amniótico. Destacam-se as semiquantitativas, tais como o indice de líquido amniótico (ILA) ${ }^{7}$ ou a medida do maior bolsão de $\mathrm{LA}^{8}$, que superaram sobremaneira as técnicas exclusivamente clínicas. A avaliação do volume de líquido amniótico pela técnica do ILA foi descrita inicialmente por Phelan et al. ${ }^{7}$, sendo denominada de técnica dos quatro quadrantes. Este método mostrou ser de fácil realização e padronização, com boa reprodutibilidade. O ILA de valor inferior ou igual a cinco centímetros caracteriza o diagnóstico de oligoidrâmnio; ao passo que valores inferiores a $3,0 \mathrm{~cm}$ caracterizam oligoidrâmnio grave .

Com base na importância do diagnóstico do oligoidrâmnio, particularmente nas gestações de alto risco, o acompanhamento evolutivo do volume de líquido amniótico é considerado um dos passos fundamentais para adequada propedêtica da vitalidade fetal.

Embora a literatura relate a associação entre o diagnóstico de oligoidrâmnio e resultados perinatais adversos ${ }^{10-12}$, persistem controvérsias quanto aos valores do ILA que seriam capazes de discriminar gestações que necessitam de atuação mais agressiva e intervencionista, principalmente quando este diagnóstico ocorre longe do termo.

Esta situação, geradora de inconsistência nos diversos protocolos de conduta já estabelecidos, estimula a busca de subsídios que possam delinear, de forma clara, esta questão. Com este intuito, o presente estudo objetiva analisar comparativamente resultados perinatais e dos testes de avaliação da vitalidade fetal em dois grupos de gestantes de alto risco, estabelecidos de acordo com o diagnóstico de oligoidrâmnio grave e não grave.

\section{Pacientes e Métodos}

Foram analisados retrospectivamente 572 casos de gestações simples com diagnóstico de oligoidrâmnio, caracterizado por ILA de valor igual ou inferior a cinco centímetros. Não foram incluídos casos que apresentassem diagnóstico de gestações múltiplas, anomalias fetais e rotura prematura de membranas. Todas as pacientes foram avaliadas pelo Setor de Vitalidade Fetal e internadas na Clínica Obstétrica do Hospital das Clínicas da Faculdade de Medicina da Universidade de São Paulo (HC-FMUSP).

A média da idade das grávidas foi de 27,5 anos, variando de 17 a 42 anos e com desvio-pa- drão de 6,7 anos. Duzentos e nove pacientes eram primigestas $(36,5 \%)$ e o restante apresentava duas ou mais gestações anteriores. Cada paciente foi avaliada quanto ao bem estar fetal pela realização dos seguintes exames propedêuticos: cardiotocografia, perfil biofisico fetal (PBF) e dopplervelocimetria obstétrica.

A cardiotocografia (CTG) anteparto de repouso foi realizada por um periodo de, no mínimo, 15 minutos, com a paciente em posição de semiFowler. Foram utilizados aparelhos da marca Hewlett Packard, modelo HP 8041 ${ }^{\mathrm{A}}$ e HP50A, com velocidade de registro gráfico de um centímetro por minuto. A análise do traçado baseou-se no índice cardiotocométrico de Zugaib e Behle ${ }^{6}$, sendo o feto classificado como ativo (normal), hipoativo (suspeito) ou inativo (alterado com desacelerações).

$\mathrm{O}$ PBF associou o estudo dos seguintes parâmetros biofísicos fetais: freqüência cardiaca fetal (FCF) analisada pela CTG, movimentos respiratórios, movimentos corpóreos, tônus e volume de líquido amniótico. Foi utilizado aparelho de ultrasonografia da marca Toshiba SAL77B ou Diasonics SPA 1000. De acordo com a rotina adotada neste serviço $^{13}$, o PBF foi classificado conforme a sua pontuação final em: normal (8 ou 10), suspeito (6) ou alterado (4, 2 ou 0$)$.

A avaliação do volume de líquido amniótico foi efetuada pela mensuração do ILA, de acordo com a metodologia proposta por Phelan et al. ${ }^{7}$. O oligoidrâmnio foi caracterizado quando constatado ILA de valor inferior ou igual a $5,0 \mathrm{~cm}$, sendo este limite utilizado na caracterização do volume de LA anormal dentro do PBF. O ILA de valor inferior ou igual a três centímetros caracterizou o oligoidrâmnio grave.

A dopplervelocimetria foi realizada por via transabdominal, utilizando-se os seguintes equipamentos de ultra-sonografia: ATL Ultramark 9 com Doppler colorido e Diasonics SPA1000 com Doppler pulsátil. Os exames foram realizados com a paciente em posição semi-sentada. Os sonogramas dos vasos analisados foram obtidos com as imagens congeladas durante a inatividade fetal e em períodos de apnéia. Foram utilizados filtros de baixa freqüência (50 hertz) e analisados os sonogramas com ondas uniformes.

Para a obtenção do sonograma das artérias umbilicais, a insonação foi feita em alça livre de cordão. Quando evidenciadas velocidades diastólicas reduzidas, procurou-se analisar o cordão na porção próxima à inserção placentária. Foi calculada a relação sístole/diástole (A/B) e seus resultados foram classificados como alterados quando acima do percentil 95 da curva de normalidade adotada ${ }^{14}$.

A avaliação dopplervelocimétrica da artéria 
cerebral média (ACM) foi realizada em corte transversal do polo cefálico fetal, na altura dos tálamos com o transdutor deslocado obliquamente, em direção à base do crânio, evitando-se a compressão excessiva do abdome materno. Neste ponto a ACM é facilmente identificada como um ramo maior do poligono de Willis. A centralização da circulação fetal foi caracterizada por índice de pulsatilidade com valor inferior ao quinto percentil para a idade gestacional $^{15}$.

Foram analisados os seguintes resultados perinatais: tipo de parto, idade gestacional no nascimento, índices de Apgar de $1^{\circ}$ e $5^{\circ}$ minuto, $\mathrm{pH}$ da artéria umbilical no nascimento, peso do recémnascido (RN), adequação do peso do RN à idade gestacional e internação em unidade de terapia intensiva (UTI) neonatal. Os RN foram classificados como pequenos para a idade gestacional (PIG) quando o peso era inferior ao percentil 10 para a idade gestacional ${ }^{16}$.

A idade gestacional foi calculada a partir da data da última menstruação (DUM) quando esta foi compativel com exame ultra-sonográfico realizado até a $20^{\mathrm{a}}$ semana. Quando a paciente não soube referir a DUM ou na discordância com o exame ultra-sonográfico, a datação da gestação baseou-se na primeira ultra-sonografia realizada.

Foram analisadas as freqüências dos resultados dos exames para a comparação entre os grupos de estudo. Para as variáveis qualitativas utilizou-se do teste de $\chi^{2}$ com correção de Yates para continuidade e, quando pertinente, o teste exato de Fisher. Para as variáveis quantitativas, utilizou-se do teste $t$ de Student. Adotou-se como nivel de significância o valor $0,05(\alpha=5 \%)$. Com isso, níveis descritivos (p) inferiores a esse valor foram considerados significantes $(p<0,05)$.

\section{Resultados}

Dos 572 casos de oligoidrâmnio, 38,5\% (220 casos) apresentavam ILA de valor inferior ou igual a $3,0 \mathrm{~cm}$ caracterizando oligoidrâmnio grave, e $61,5 \%$ (352 casos) apresentavam ILA entre 3,1 e $5,0 \mathrm{~cm}$. A proporção de primigestas foi de $34,5 \%$ no grupo com oligoidrâmnio grave e de $37,8 \%$ nos casos com ILA entre 3,1 e $5,0 \mathrm{~cm}$. As pacientes com oligoidrâmnio grave apresentaram média de idade de 27,5 anos e desvio padrão de 6,8 anos, e as com ILA entre 3,1 e $5,0 \mathrm{~cm}$ apresentaram média de idade de 27,6 anos com desvio-padrão de 6,7 anos. Não foram observadas diferenças significativas entre as médias das idades maternas.

Na Tabela 1 podemos observar a distribuição dos resultados dos testes de avaliação da vitalidade fetal realizados no período anteparto, de acordo com a gravidade do oligoidrâmnio. Verificou-se associação significativa entre alterações na cardiotocografia no período anteparto e a ocorrência de oligoidrâmnio grave $(p<0,05)$. Realizando-se a partição do $\chi^{2}$, constatou-se que a proporção de casos com CTG suspeita foi significativamente maior no grupo com oligoidrâmnio grave $(\mathrm{p}=0,0058)$. Entretanto, não foi observada diferença significativa quanto à proporção de casos com CTG alterada $(p=0,4305)$. Houve associação significativa entre as alterações no resultado do PBF e a gravidade do oligoidrâmnio $(\mathrm{p}<0,05)$. A distribuição dos casos de acordo com o resultado do PBF também apresentou associação significativa $(\mathrm{p}<0,05)$ com o tipo de oligoidrâmnio. Realizando-se a partição do $\chi^{2}$, observa-se proporção significativamente maior de exames normais no grupo com ILA entre 3,1 e 5,0 cm ( $p=0,0058)$, e também maior proporção de exames alterados no grupo com oligoidrâmnio grave $(\mathrm{p}=0,0038)$.

Tabela 1 - Resultados dos testes de avaliação da vitalidade fetal de acordo com a gravidade do oligoidrâmnio.

\begin{tabular}{lccc}
\hline & Oligoidrâmnio & Oligoidrâmnio grave & \\
& ILA 3,1 a 5,0 cm & ILA $\leq 3,0 \mathbf{c m}$ & p \\
\hline Cardiotocografia & $\mathrm{n} . \quad \%$ & $\mathrm{n} . \quad \%$ & \\
Normal & $303(86,1)$ & $169(76,8)$ & \\
Suspeita & $32(9,1)$ & $37(16,8)$ & $\chi^{2}=8,70$ \\
Alterada & $17(4,8)$ & $14(6,4)$ & $\mathrm{p}=0,01$ \\
$\quad$ Total & 351 & 219 & \\
Perfil biofísico fetal & & & \\
Normal (8) & $225(63,9)$ & $115(52,2)$ & \\
Suspeito (6) & $112(31,8)$ & $82(37,3)$ & $\chi^{2}=12,09$ \\
Alterado (0/2/4) & $15(4,3)$ & $23(10,5)$ & $\mathrm{p}=0,002$ \\
$\quad$ Total & 350 & 218 & \\
Dopplervelocimetria da artéria umbilical & & \\
Normal & $260(81,5)$ & $151(82,1)$ & \\
Alterada & $59(18,5)$ & $33(17,9)$ & $\mathrm{NS}$ \\
Total & 319 & 184 & \\
Dopplervelocimetria da artéria cerebral média & & \\
Normal & $113(66,1)$ & $40(45,5)$ & $\chi^{2}=9,39$ \\
Alterada & $58(33,9)$ & $48(54,5)$ & $\mathrm{p}=0,002$ \\
Total & 171 & 88 &
\end{tabular}

Os dados da dopplervelocimetria obstétrica, demonstrados na Tabela 1, revelam que não houve associação entre a gravidade do oligoidrâmnio e os resultados de acordo com a análise da relação A/B das artérias umbilicais. Entretanto, a distribuição dos resultados da dopplervelocimetria da $\mathrm{ACM}$ demonstra associação significativa $(\mathrm{p}=0,002)$ 
entre o oligoidrâmnio grave e a ocorrência de exames alterados caracterizando a centralização da circulação fetal.

$\mathrm{Na}$ análise dos resultados perinatais, observou-se que, em relação à idade gestacional e ao peso no nascimento, não foram constatadas diferenças significativas nas médias, de acordo com a gravidade do oligoidrâmnio (Tabela 2). Verificouse que a proporção de casos com líquido amniótico meconial foi significativamente maior $(p<0,05)$ no grupo com oligoidrâmnio grave. Nos casos em que foi possivel avaliar o $\mathrm{pH}$ no nascimento, não foram caracterizadas diferenças significativas quanto à gravidade do oligoidrâmnio.

Tabela 2 - Resultados neonatais de acordo com a gravidade do oligoidrâmnio.

\begin{tabular}{|c|c|c|c|}
\hline Resultados neonatais & $\begin{array}{l}\text { Oligoidrâmnio } \\
\text { ILA 3,1 a 5,0cm }\end{array}$ & $\begin{array}{l}\text { Oligoidrâmnio grave } \\
\text { ILA } \leq 3,0 \mathrm{~cm}\end{array}$ & $\mathrm{p}$ \\
\hline \multicolumn{4}{|c|}{ Peso do recém-nascido (gramas) } \\
\hline Média & 2725,4 & 2600,5 & NS \\
\hline Desvio-padrão & 741,7 & 771,0 & \\
\hline \multicolumn{4}{|c|}{ Idade gestacional no nascimento (semanas) } \\
\hline Média & 37,8 & 37,8 & NS \\
\hline Desvio-padrão & 3,2 & 3,3 & \\
\hline \multicolumn{4}{|c|}{ Mecônio no líquido amniótico } \\
\hline Sim & $46(16,8 \%)$ & $41(27,9 \%)$ & \\
\hline Não & $227(83,2 \%)$ & $106(72,1 \%)$ & $p<0,05$ \\
\hline Total & 273 & 147 & \\
\hline \multicolumn{4}{|l|}{ pH no nascimento } \\
\hline Média & 7,252 & 7,231 & NS \\
\hline Desvio-padrão & 0,092 & 0,134 & \\
\hline $\mathrm{pH}<7,20$ & $51(23,3 \%)$ & $37(28,3 \%)$ & \\
\hline $\mathrm{pH} \geq 7,20$ & $168(76,7 \%)$ & $94(71,8 \%)$ & NS \\
\hline Total & 219 & 131 & \\
\hline
\end{tabular}

A Figura 1 mostra os resultados neonatais nos casos de oligoidrâmnio, e observa-se que a proporção de recém-nascidos PIG foi significativamente maior $(\mathrm{p}<0,05)$ nos casos com oligoidrâmnio grave $(32,7 \%)$ quando comparado aos casos com ILA entre 3,1 e $5,0 \mathrm{~cm}(21,0 \%)$. Os demais resultados neonatais não apresentaram diferenças significativas de acordo com a gravidade do oligoidrâmnio.

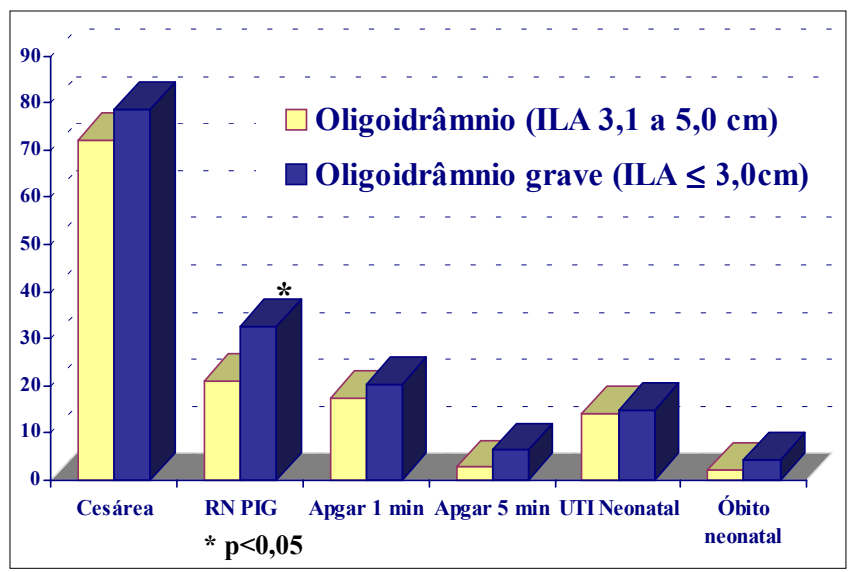

Figura 1 - Resultados perinatais de acordo com a gravidade do oligoidrâmnio.

\section{Discussão}

A avaliação do volume de LA é de fundamental importância nas gestações de alto risco, uma vez que a sua diminuição progressiva permite identificar os fetos que apresentam maior risco para o sofrimento fetal ${ }^{1,5,8,10,12}$. Em trabalho realizado nesta Instituição ${ }^{2}$ no qual se analisou 524 gestações de alto risco, observou-se que o sofrimento fetal intraparto ou anteparto esteve presente em $26 \%$ dos casos com oligoidrâmnio (ILA inferior ou igual a $5,0 \mathrm{~cm}$ ). Outros estudos também relatam a associação entre o oligoidrâmnio e resultados perinatais adversos, principalmente o sofrimento fetal e a ocorrência de desacelerações da freqüência cardíaca fetal ${ }^{2,17-19}$.

Apesar de os dados da literatura concordarem que o oligoidrâmnio, caracterizado pelo ILA inferior ou igual a cinco, identifique fetos de risco para resultados perinatais adversos, alguns autores questionam esta associação, principalmente em gestações de termo sem complicações. Magann et $a .^{20}$ não constatam esta associação e, 
em gestações de alto risco, questionam se a definição adotada para oligoidrâmnio é realmente adequada. Glantz e D'Amico ${ }^{21}$ não observaram associação entre oligoidrâmnio e a presença de desacelerações variáveis em casos com a cardiotocografia reativa. Deve-se ressaltar que, no estudo de Magann et $a .^{20}$, a amnioinfusão foi um dos procedimentos adotados para casos de sofrimento fetal, visando corrigir o volume de líquido amniótico para reduzir a ocorrência de fenômenos compressivos do funículo. Esta conduta pode contribuir para a não constatação de significativa associação entre resultados adversos e o oligoidrâmnio.

Apesar de alguns autores criticarem a conduta resolutiva adotada para casos de oligoidrâmnio, o fato de não serem observados resultados negativos pode indicar que a resolução da gestação seja a medida mais adequada por não produzir repercussões ao produto conceptual. Para que esta questão seja definitivamente esclarecida, são necessários estudos randomizados, comparandose diferentes protocolos clínicos para os casos com diagnóstico de oligoidrâmnio.

O presente estudo teve como meta aprofundar os conhecimentos acerca dessa intercorrência obstétrica, separando os casos em dois grupos segundo o valor do ILA. O limite arbitrário adotado de $3,0 \mathrm{~cm}$ constitui um valor que, na prática, tem se mostrado útil na condução desses casos, notadamente quando outras evidências indicam vantagens em se postergar a interrupção da gravidez. Alguns autores preconizam a associação de outros exames para se evidenciar grupos de maior gravidade. Carroll e Bruner ${ }^{22}$ relatam que valores elevados na relação A/B da dopplervelocimetria da artéria umbilical identificam os casos de maior risco para resultados perinatais adversos. Scott et al. ${ }^{23}$, referem que a dopplervelocimetria da artéria cerebral média pode contribuir na verificação dos fetos de risco para resultados adversos. Miyadahira et al. ${ }^{24}$ observaram que a análise conjunta da cardiotocografia e da dopplervelocimetria da artéria cerebral média em casos de oligoidrâmnio auxilia na detecção dos casos de maior gravidade, e conseqüentemente com pior resultado perinatal.

O diagnóstico de oligoidrâmnio, quando fruto da adaptação cardiocirculatória fetal a um estresse provocado pela hipoxemia crônica, pode ou não estar acompanhado de alterações agudas da vitalidade fetal. Os fenômenos adaptativos fetais, ao proporcionarem condições que favoreçam a oxigenação fetal, oferecem melhores condições para que o recém-nascido não apresente alterações importantes dos índices de Apgar ou dos valores do $\mathrm{pH}$ no nascimento.

A ocorrência mais freqüente de líquido amniótico meconial no grupo grave é evento esperado nesta perspectiva, pois a eliminação de mecônio está relacionada à compressão do cordão umbilical, cuja vulnerabilidade é, evidentemente, mais acentuada na oligoidramnia grave. Da mesma forma, a maior incidência de recémnascidos PIG apresenta-se em consonância com a maior gravidade do oligoidrâmnio, embora não se constate diferenças entre os grupos quanto à idade gestacional no nascimento e o peso dos recém-nascidos.

Os resultados da presente pesquisa permitem afirmar que a divisão das gestações com oligoidrâmnio em dois grupos, de acordo com a gravidade da redução do volume de líquido amniótico, associa-se a alterações nos exames antenatais da vitalidade fetal, e com a desnutrição fetal (recémnascidos PIG). Todavia, excetuando-se a presença de líquido amniótico meconial, os resultados neonatais não diferem em função da gravidade do oligoidrâmnio. Infere-se, portanto, a adequada condução dos casos em questão, já que o aspecto mais importante nos cuidados às gestações de alto risco com oligoidrâmnio vincula-se aos resultados finais.

Em conclusão, as observações deste estudo constatam que, a caracterização da gravidade do oligoidrâmnio permite discriminar, nas gestações de alto-risco, os casos que se associam a pior resultado perinatal. A adoção de conduta conservadora em casos de oligoidramnia que necessitem de postergação para a resolução da gestação, só encontra algum respaldo nos casos em que o oligoidrâmnio não é caracterizado como grave, sendo imprescindivel a realização dos demais exames de avaliação da vitalidade fetal.

\section{ABSTRACT}

Purpose: to evaluate, in the high-risk pregnancies with oligohydramnios, the assessment tools for fetal well-being and perinatal results.

Methods: five hundred seventy-two high-risk pregnancies were retrospectively analyzed. All of them presented with oligohydramnios established by AFI $\leq 5.0 \mathrm{~cm}$. Severe oligohydramnios was detected in 220 cases $(A F I \leq 3,0 \mathrm{~cm})$. The fetal well-being tests included: antepartum cardiotocography, biophysical profile score (BPS) and dopplervelocimetry of umbilical and middle cerebral arteries. Multiple gestation, fetal anomalies and premature rupture of membrane cases were excluded.

Results: severe oligohydramnios was significantlly associated with abnormal and suspected cardiotocography results (23.2\%), abnormal biophysical profile score (10.5\%), abnormal results of middle cerebral artery dopplervelocimetry (54.5\%), small for gestational age infants 
(32.7\%) and meconial amniotic fluid (27.9\%) when compared to pregnancies with AFI between 3.1 and $5.0 \mathrm{~cm}$. This group presented: abnormal or suspected cardiotocography results (13.9\%), abnormal biophysical profile score (4.3\%), abnormal results of middle cerebral artery dopplervelocimetry (33.9\%), small for gestational age infants (21.0\%) and meconial amniotic fluid (16.8\%).

Conclusion: the oligohydramnios severity in high-risk pregnancies allows to discriminate the cases that are related to adverse perinatal outcome.

KEY WORDS: Amniotic fluid. Oligohydramnios. High-risk pregnancies. Perinatal results.

\section{Referências}

1. Philipson EH, Sokol RJ, Williams T. Oligohydramnios: clinical associations and predictive value for intrauterine growth retardation. Am J Obstet Gynecol 1983; 146:271-8.

2. Yamamoto RM. Oligoidrâmnio: correlação com a vitalidade fetal e resultados perinatais [dissertação]. São Paulo: Universidade de São Paulo; 1995.

3. Rezende J, Montenegro CAB. Trocas maternoovulares. In: Rezende J, editor. Obstetrícia. 9 ${ }^{a}$ ed. Rio de Janeiro: Guanabara Koogan; 2002. p.83-95.

4. Berhman RE, Lees MH, Peterson EN, De Lannoy $\mathrm{CW}$, Seeds AE. Distribution of the circulation in the normal and asphyxiated fetal primate. Am J Obstet Gynecol 1970; 108:956-69.

5. Hoskins IA, Frieden FJ, Young BK. Variable decelerations in reactive nonstress tests with decreased amniotic fluid index predict fetal compromise. Am J Obstet Gynecol 1991;165:1094-8.

6. Zugaib M, Behle I. Monitoração fetal eletrônica. $1^{\text {a }}$ ed. São Paulo: Roca; 1981.

7. Phelan JP, Smith CV, Broussard P, Small M. Amniotic fluid volume assessment with the four-quadrant technique at 36-42 weeks' gestation. J Reprod Med 1987 ; 32:540-2.

8. Chamberlain PF, Manning FA, Morrison I, Harman CR, Lange IR. Ultrasound evaluation of amniotic fluid volume. II. The relationship of increased amniotic fluid volume to perinatal outcome. Am J Obstet Gynecol 1984; 150:250-4.

9. Nomura RMY, Francisco RPV. Líquido amniótico. In: Okumura M, Zugaib M, editores. Ultra-sonografia em Obstetrícia. $1^{\text {a }}$ ed. São Paulo: Sarvier; 2002. p.109-26.

10.Casey BM, McIntire DD, Bloom SL, et al. Pregnancy outcomes after antepartum diagnosis of oligohydramnios at or beyond 34 weeks' gestation. Am J Obstet Gynecol 2000; 182:909-12.
11.Chauhan SP, Sanderson M, Hendrix NW, Magann EF, Devoe LD. Perinatal outcome and amniotic fluid index in the antepartum and intrapartum periods: a meta-analysis. Am J Obstet Gynecol 1999; 181:1473-8.

12.Sarno AP Jr, Ahn MO, Brar HS, Phelan JP, Platt LD. Intrapartum doppler velocimetry, amniotic fluid volume, and fetal heart rate as a predictors of subsequent fetal distress. I. An initial report. Am J Obstet Gynecol 1989; 161:1508-14.

13.Yamamoto RM, Francisco RPV, Miyadahira S, Zugaib M. Perfil biofísico fetal modificado: aspectos atuais na padronização do método. Rev Ginecol Obstet 2000; 11:118-22.

14.Amim Júnior J. Dopplerfluxometria da artéria umbilical. Valores normais e importância no diagnóstico do crescimento intra-uterino retardado na gestação de alto risco [dissertação]. Rio de Janeiro: Universidade Federal do Rio de Janeiro; 1989.

15.Arduini D, Rizzo G. Normal values of pulsatility index from fetal vessels: a cross-sectional study on 1556 healthy fetuses. J Perinat Med 1990; 18:165-72.

16. Ramos JLA. Avaliação do crescimento intra-uterino por medidas antropométricas do recém-nascido [tese]. São Paulo: Universidade de São Paulo; 1983.

17.Hadar A, Sheiner E, Hallak M, Katz M, Mazor M, Shoham-Vardi I. Abnormal fetal heart rate tracing patterns during the first stage of labor: effect on perinatal outcome. Am J Obstet Gynecol 2001; 185:863-8.

18.Rainford M, Adair R, Scialli AR, Ghidini A, Spong CY. Amniotic fluid index in the uncomplicated term pregnancy: prediction of outcome. J Reprod Med 2001; 46:589-92.

19. Oron T, Sheiner E, Shoham-Vardi I, Mazor M, Katz M, Hallak M. Risk factors for antepartum fetal death. J Reprod Med 2001; 46:825-30.

20.Magann EF, Kinsella MJ, Chauhan SP, McNamara MF, Gehring BW, Morrison JC. Does an amniotic fluid index $£ 5 \mathrm{~cm}$ necessitate delivery in high-risk pregnancies? A case-control study. Am J Obstet Gynecol 1999; 180:1354-9.

21.Glantz C, D'Amico ML. Lack of relationship between variable decelerations during reactive nonstress tests and oligohydramnios. Am J Perinatol 2001; 18:129-35.

22. Carroll BC, Bruner JP. Umbilical artery Doppler velocimetry in pregnancies complicated by oligohydramnios. J Reprod Med 2000; 45:562-6.

23. Scott LL, Casey BM, Roberts S, McIntire D, Twickler DM. Predictive value of serial middle cerebral and renal artery pulsatility indices in fetuses with oligohydramnios. J Matern Fetal Med 2000; 9:105-9.

24.Miyadahira S, Yamamoto RM, Francisco RPV, Zugaib M. Dopplerfluxometria da artéria cerebral média em gestações com oligoidrâmnio. Rev Ginecol Obstet 1996; 7:111-7. 